\title{
The antioxidant effect of dietary phytoestrogens in the prevention of osteocyte apoptosis - implications for bone health in ageing
}

\author{
K. Rado, B. Noble and V. Mann \\ School of Science, Technology and Health, University Campus Suffolk, Ipswish, IP41QJ, UK
}

The accumulation of oxidative damage, caused by increased levels of reactive oxygen species (ROS) during the process of ageing has been suggested to be involved in the development of age-related bone loss ${ }^{(1)}$. In women withdrawal of estrogen represents the primary factor determining post-menopausal bone loss and has been associated with negative indicators of bone quality with increased age including the apoptotic death of osteocytes in vivo ${ }^{(2)}$. Estrogen has been shown to be effective in the reduction of osteocyte apoptosis via estrogen receptor mediated mechanism. More recently estrogen has been shown to protect bone cells from ROS attack via indirect antioxidant mechanisms ${ }^{(3)}$. Phytoestrogens are naturally occurring dietary compounds that are structurally similar to estrogen and there is growing evidence of the beneficial effects of phytoestrogens in the maintenance of bone health ${ }^{(4)}$. The aim of this study was to investigate the potential anti-oxidant effects of phytoestrogens in the prevention of oxidative stress induced apoptosis in osteocytes in vitro.

Oxidative stress was induced in the osteocyte like cell line MLO-Y4 using treatment with $\mathrm{H}_{2} \mathrm{O}_{2}$. Apoptosis was determined in MLO-Y4 osteocytes based on the morphological changes characteristic to apoptotic cells including rounding up of the cell, plasma membrane blebbing, nuclear and cytoplasmic condensation, and nuclear fragmentation. The protective effects of two soya derived phytoestrogens Genistein and Daidzein were determined by pre-treatment of MLO-Y4 with either compound prior to induction of oxidative stress with $\mathrm{H}_{2} \mathrm{O}_{2}$.

A 2 hour treatment of MLO-Y4 with $0.4 \mathrm{mM} \mathrm{H}_{2} \mathrm{O}_{2}$ significantly induced apoptosis in MLO-Y4 cultures when compared to control $(p<0.001)$. The percentage of $\mathrm{H}_{2} \mathrm{O}_{2}$ induced apoptotic osteocytes was significantly reduced after pre-treatment of cells for 1 hour with Genistein at $10^{-9} \mathrm{M}(p<0.001)$ or with Daidzein at $10^{9} \mathrm{M}(p<0.001)$.

It is possible that the loss of osteocytes associated with the ageing process may occur through a failure to suppress the activity of naturally occurring or disease associated oxidant molecules. These data suggest that the osteocyte protective effects of phytoestrogens may operate in a similar manner to estrogen and may provide evidence in the support of a potential bone sparing effect for these dietary compounds.

1. Finkl T \& Holbrook NJ (2000) Nature 408, 239-247.

2. Tomkinson A, Reeve J, Shaw RW et al. (1997) J.Clin Endocrinol Metab 222, 236-245.

3. Mann V, Huber C, Kogianni G et al. (2007) Bone 40, 674-684.

4. Coxam V (2008) Proceedings of the Nutrition Society 67, 184-195. 